\title{
3-Substituted and 2,3-Disubstituted Cyclopentanones via an Asymmetric Tandem 1,4-Addition/Dieckmann Cyclization ${ }^{1}$
}

\author{
Ulrich Groth,* Wolfgang Halfbrodt, Aris Kalogerakis, Thomas Köhler, Paul Kreye \\ Fachbereich Chemie der Universität Konstanz, Universitätsstrasse 10, Postfach M-720, 78457 Konstanz, Germany \\ Fax +49(7531)884155; E-mail: ulrich.groth@uni-konstanz.de
}

\begin{abstract}
A new stereoselective method for the synthesis of 3-substituted and 2,3-disubstituted cyclopentanones is described. The key step is the 1,4-addition of a cuprate to a chilar Michael-acceptor derived from (-)-8-phenylmenthol or the Helmchen auxiliary followed by Dieckmann cyclization of the obtained chiral enolates. The resultant 2,3-cyclopentanones can be transformed after methanolysis and demethoxycarbonylation to the related 3-substituted cyclopentanones.
\end{abstract}

Key words: asymmetric synthesis, cyclopentanones, cuprate, chiral auxiliary, 1,4-addition, Dieckmann cyclization, natural products

2,3-Disubstituted and 2,2',3-trisubstituted cyclopentanones such as jasmonic acid and its related compounds, ${ }^{2}$ chokol A, ${ }^{3}$ sarkomycin, ${ }^{4}$ dactylol, ${ }^{5}$ confertin, ${ }^{6}$ 11deoxyprostaglandines ${ }^{7}$ and steroids ${ }^{8}$ are attractive target molecules in organic synthesis due to their biological activity and pharmaceutical importance. Consequently, a number of methods have been applied for the asymmetric construction of cyclopentanones. ${ }^{9}$ Since some conjugate addition/cyclization reactions have been reported ${ }^{10}$ in connection with our own studies in steroid synthesis ${ }^{11}$ we have developed a versatile applicable method for the asymmetric synthesis of 3-substituted-2-carbomethoxyand 3-substituted-cyclopentanones. We were able to synthesize two different chiral enoates $\mathbf{5 a}$ and $\mathbf{5 b}$, which allowed the conjugate addition of various organocopper compounds. The intermediates 6 of this addition led via an intramolecular cyclization (Dieckmann cyclization) to 2,3-disubstituted cyclopentanones 7 in good yields and with high diastereoselectivity (Scheme 1). These gave upon methanolysis the enantiopure 2-carbomethoxycyclopentanones 8 (Scheme 2), which can be used as starting materials in the synthesis of natural products containing a cyclopentane moiety.

The (-)-8-phenylmenthol-derived Michael-acceptor 5a was prepared in a Wittig-Horner reaction from methyl-4oxo-butanoate $\mathbf{4}$ and the diethoxy phosphonic ester $\mathbf{3 a}$ in $87 \%$ yield; as a by-product the corresponding cis-isomer of $\mathbf{5 a}$ was isolated in $4 \%$ yield. Compound 4 was obtained in two steps by an improved synthesis according to Saji. ${ }^{12}$ Compund 3a was prepared in a Michael-Arbuzov reaction from the chloroacetate $\mathbf{2 a}$, an intermediate in the syn- thesis of (-)-8-phenylmenthol. ${ }^{13}$ The other chiral acceptor 5b was synthesized in a similar way starting from $\mathbf{1}$ and alcohol $\mathbf{B}$ - the Helmchen auxiliary - in $51 \%$ yield over 3 steps. While reaction of the organocuprates $\mathrm{RCu}-$ $\mathrm{Li} \cdot n \mathrm{Bu}_{3} \cdot \mathrm{PBF}_{3}$, which were prepared according to Oppolzer ${ }^{14}$ from MeLi or PhLi, copper(I)iodide, tributyl phosphine and $\mathrm{BF}_{3} \cdot \mathrm{Et}_{2} \mathrm{O}$, with $\mathbf{5 a}$ gave the cyclopentanones $\mathbf{7} \mathbf{a}$ and $\mathbf{7 b}$ in goods yields and with high diastereoselectivity, the analogue organocuprate $t$-BuCuLi$\cdot n$ $\mathrm{Bu}_{3} \mathrm{P} \cdot \mathrm{BF}_{3}$ generated from $t$-BuLi did not react (Table 1, entry 3 ). We were able to synthesize the cyclopentanone 7c by changing from copper(I)iodide to copper(I)cyanide. ${ }^{15}$ In this case the use of the tributyl phosphine is not necessary, which makes the purification of the synthesized cyclopentanone by column chromatography easier. Next, we studied the reaction of allylcuprates with $\mathbf{5 a}$ by choosing three different cuprate adducts, but unfortunately the 3-allyl-substitued cyclopentanone $\mathbf{7 d}$ could not be obtained. In the course of our synthetic studies towards

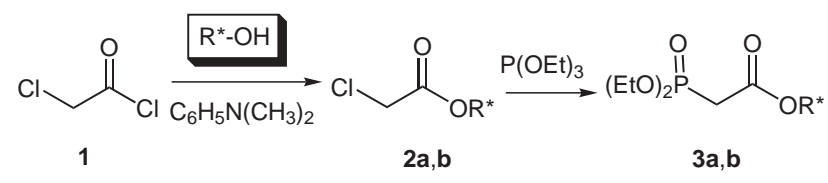

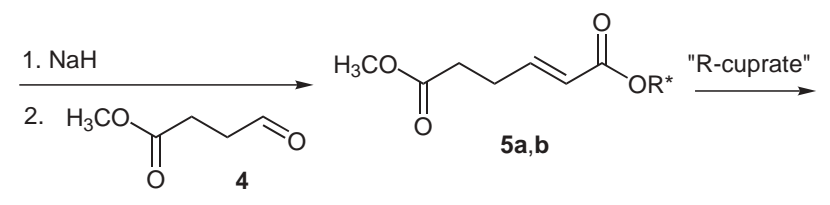<smiles>[R]C(/C=C(\[O-])O[I+])CCC(=O)OC</smiles>

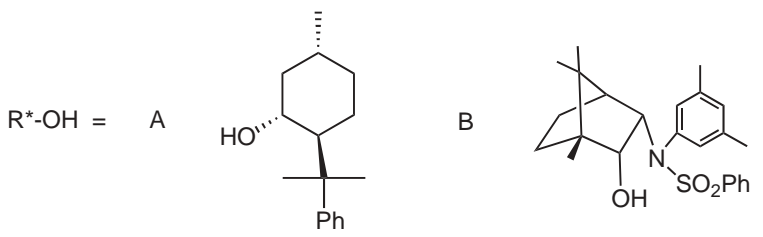

Scheme 1 
Table 1 1,4-Addition/Dieckmann Cyclization of Cuprates with the Chiral Acceptors ${ }^{17}$

\begin{tabular}{|c|c|c|c|c|c|}
\hline Entry & $\mathrm{R}$ & $5, \mathrm{R}^{*}$ & Cuprate & $7(\%)$ & de $(\%)^{\mathrm{a}}$ \\
\hline 1 & $\mathrm{CH}_{3}$ & A & $\mathrm{RCuLi} \cdot n-\mathrm{Bu}_{3} \mathrm{P} \cdot \mathrm{BF}_{3}$ & $7 \mathbf{a}(79)$ & 78 \\
\hline 2 & $\mathrm{Ph}$ & A & $\mathrm{RCuLi} \cdot n-\mathrm{Bu}_{3} \mathrm{P} \cdot \mathrm{BF}_{3}$ & $7 b(67)$ & 76 \\
\hline 3 & $t$-Bu & A & $\mathrm{RCuLi} \cdot n-\mathrm{Bu}_{3} \mathrm{P} \cdot \mathrm{BF}_{3}$ & - & - \\
\hline 4 & $t-\mathrm{Bu}$ & A & $\mathrm{R}_{2} \mathrm{Cu}(\mathrm{CN}) \mathrm{Li}_{2} \cdot \mathrm{BF}_{3}$ & $7 c(85)$ & 75 \\
\hline 5 & $\mathrm{CH}_{2}=\mathrm{CH}_{2} \mathrm{CH}_{2}$ & A & $\mathrm{RCuLi} \cdot n \mathrm{Bu}_{3} \mathrm{P} \cdot \mathrm{BF}_{3}$ & - & - \\
\hline 6 & $\mathrm{CH}_{2}=\mathrm{CH}_{2} \mathrm{CH}_{2}$ & A & $\mathrm{R}_{2} \mathrm{CuLi}_{2} \cdot n-\mathrm{Bu}_{3} \mathrm{P} \cdot \mathrm{BF}_{3}$ & - & - \\
\hline 7 & $\mathrm{CH}_{2}=\mathrm{CH}_{2} \mathrm{CH}_{2}$ & A & $\mathrm{R}_{2} \mathrm{Cu}(\mathrm{CN}) \mathrm{Li}_{2} \cdot \mathrm{BF}_{3}$ & - & - \\
\hline 8 & $\mathrm{CH}_{2}=\mathrm{CH}$ & A & $\mathrm{RCuLi} \cdot n \mathrm{Bu}_{3} \mathrm{P} \cdot \mathrm{BF}_{3}$ & - & - \\
\hline 9 & $\mathrm{CH}_{2}=\mathrm{CH}$ & A & $\mathrm{R}_{2} \mathrm{CuLi}_{2} \cdot n \mathrm{Bu}_{3} \mathrm{P} \cdot \mathrm{BF}_{3}$ & $7 e(78)$ & 82 \\
\hline 10 & $\mathrm{CH}_{2}=\mathrm{CH}$ & $\mathrm{B}$ & $\mathrm{R}_{2} \mathrm{Cu}(\mathrm{CN}) \mathrm{Li}_{2} \cdot \mathrm{BF}_{3}$ & $\mathbf{7 f}(79)$ & 78 \\
\hline 11 & $\mathrm{CH}_{2}=\mathrm{CH}$ & B & $\mathrm{R}_{2} \mathrm{Cu}(\mathrm{CN}) \mathrm{Li}_{2} \cdot \mathrm{BF}_{3}{ }^{\mathrm{b}}$ & $7 \mathbf{f}(88)$ & 89 \\
\hline 12 & $\mathrm{CH}_{2}=\mathrm{CH}$ & $\mathrm{B}$ & $\mathrm{R}_{2} \mathrm{Cu}(\mathrm{CN}) \mathrm{Li}_{2} \cdot \mathrm{BF}_{3}{ }^{\mathrm{c}}$ & $\mathbf{7 f}(78)$ & 89 \\
\hline
\end{tabular}

${ }^{\mathrm{a}}$ Determined by ${ }^{13} \mathrm{C}$ NMR spectroscopy.

${ }^{b}$ The cuprate was added at $-95^{\circ} \mathrm{C}$.

${ }^{\mathrm{c}}$ The cuprate was added at $-116^{\circ} \mathrm{C}$.

the sesquiterpenes chokols we investigated the synthesis of the 2-carbomethoxy-3-vinyl-cyclopentanone $\mathbf{8 e}$ (Scheme 2, Table 2, entries 4 and 5) starting from the previous using chiral acceptor $\mathbf{5} \mathbf{a}$ and the camphor derivative $\mathbf{5 b}$ by reaction with a vinylcuprate. The Oppolzer organocuprate $\left(\mathrm{CH}_{2}=\mathrm{CH}\right) \mathrm{CuLi} \cdot n-\mathrm{Bu}_{3} \mathrm{P} \cdot \mathrm{BF}_{3}$ generated from vinyllithium ${ }^{16}$ did not react with $\mathbf{5 a}$, but the reaction of the cuprate $\left(\mathrm{CH}_{2}=\mathrm{CH}\right)_{2} \mathrm{CuLi}_{2} \cdot n-\mathrm{Bu}_{3} \mathrm{P} \cdot \mathrm{BF}_{3}$ yielded $78 \%$ of the 3-vinyl- $\beta$-keto-ester $7 \mathrm{e}$ with $82 \%$ de. Similarly, the chiral enoate $\mathbf{5 b}$ derived from the Helmchen auxiliary, ${ }^{9 b}$ after reaction with vinylcuprates also gave the vinylcyclopentanone 7f in good yields and with high diastereoselectivity. Indeed, addition of $\left(\mathrm{CH}_{2}=\mathrm{CH}\right)_{2} \mathrm{Cu}(\mathrm{CN}) \mathrm{Li}_{2} \cdot \mathrm{BF}_{3}{ }^{15}$ at $-85{ }^{\circ} \mathrm{C}$ to $\mathbf{5 b}$ afforded $\mathbf{7 f}$ in $79 \%$ yield and with $78 \%$ de. We also observed that the yield and the diastereomeric excess of $7 \mathbf{f}$ can be increased ( $88 \%$ yield and $89 \%$ d.e.) by addition of the cuprate at $-95{ }^{\circ} \mathrm{C}$. However, addition at lower temperatures $(-116$ ${ }^{\circ} \mathrm{C}$ ) had no effect on the de, but the yield decreased to $78 \%$.

Methanolysis of the cyclopentanones 7 at $120{ }^{\circ} \mathrm{C}$ in a sealed tube afforded the 2-carbomethoxycyclopentanones $\mathbf{8}$ in $86-95 \%$ ee and, in addition, enantiomerically pure (-)-8-phenylmenthol $\mathbf{A}$ or camphor alcohol $\mathbf{B}$, the chiral auxiliaries, in 83-95\% yield. Obviously, the major diastereomers 7 undergo a methanolysis much faster than their C-3 epimers, so that the $\beta$-keto esters $\mathbf{8}$ can be obtained virtually in enantiomerically pure form. As a byproduct of the methanolysis an almost 1:1 mixture of 7 and its epimers was obtained in 6-11\%. The enantiomeric excess and the absolute configuration of 8a at C-3 were determined by comparison of its optical rotation with an authentic sample prepared from $(R)$-pulegone. ${ }^{18}$ The absolute configuration of $\mathbf{8} \mathbf{b}^{19}$ and $\mathbf{8} \mathbf{c}^{20}$ was determined by comparison of the optical rotation of the prepared 3-substituted-cyclopentanones 9 with the reported value. For the determination of the absolute configuration of $8 \mathbf{e}$ we compared the optical rotation of our 2-carbomethoxy-3vinylcyclopentanone $8 \mathbf{e}^{17}$ with the reported value of the same compound $\left([\alpha]_{\mathrm{D}}{ }^{20}=+85.05\right)$ synthesized from Helmchen and co-workers. ${ }^{9 \mathrm{~b}}$ The enantiomeric excess of the cyclopentanones $\mathbf{8 b}-\mathbf{e}$ and $\mathbf{9 b}-\mathbf{e}$ was obtained by determination of the diastereomeric excess of the diastereomeric ketals 10 by analysis of their ${ }^{13} \mathrm{C}$ NMR spectra. ${ }^{21}$ These were prepared after demethoxycarbonylation of $\mathbf{8}$ with $\mathrm{DABCO}^{22}$ (1,4-diazabicyclo[2.2.2] octane) in DMSO and reaction of the obtained cyclopentanones 9 with (2R,3R)-(-)-2,3-butanediol 11.
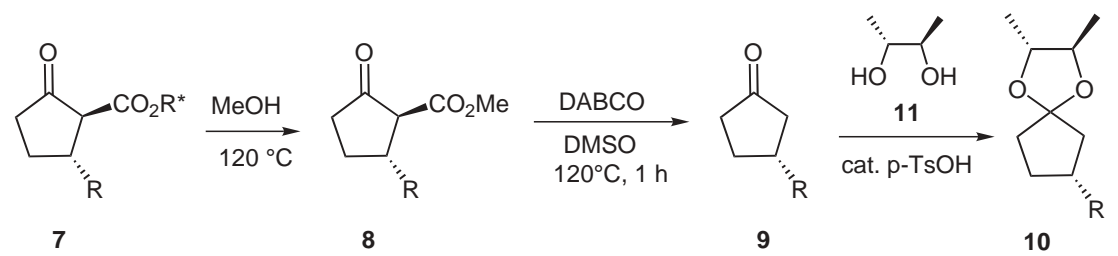

Scheme 2 
Table 2 Synthesis of the 3-Substituted Cyclopentanones

\begin{tabular}{|c|c|c|c|c|c|c|}
\hline Entry & $7, \mathrm{R}$ & $8(\%)$ & $\mathrm{R} * \mathrm{OH}(\%)$ & ee $(\%)$ & $9(\%)$ & $10(\%)$ \\
\hline 1 & $7 \mathbf{a}, \mathrm{CH}_{3}$ & $\mathbf{8 a}(84)$ & $\mathbf{A}(84)$ & 89 & - & - \\
\hline 2 & $7 \mathbf{b}, \mathrm{Ph}$ & $\mathbf{8 b}(91)$ & $\mathbf{A}(95)$ & $>95^{b}$ & 76 & 96 \\
\hline 3 & $7 \mathbf{c}, t-\mathrm{Bu}$ & $8 c(60)$ & $\mathbf{A}(92)$ & 86 & 89 & 84 \\
\hline 4 & 7e, $\mathrm{CH}_{2}=\mathrm{CH}$ & $8 \mathbf{e}(77)$ & $\mathbf{A}(83)$ & $>95^{\mathrm{b}}$ & 85 & 90 \\
\hline 5 & 7f, $\mathrm{CH}_{2}=\mathrm{CH}^{\mathrm{a}}$ & $\mathbf{8 e}(78)$ & B (86) & $>95^{b}$ & 88 & 94 \\
\hline
\end{tabular}

a Table 1, entry 11 .

${ }^{\mathrm{b}}$ Only one diastereomer was detected.

In summary, we have developed a new access to substituted cyclopentanones by employing a tandem reaction of cuprates with chiral Michael acceptors via an asymmetric 1,4-addition and a Dieckmann cyclization. The prepared 2,3-substituted cyclopentanones were then transformed into the related enantiopure 2-carbomethoxycyclopentanones and 3-substituted cyclopentanones. The synthesis of cyclopentanoid natural products using this new methodology is currently under investigation.

\section{Acknowledgment}

The authors are grateful to the Fonds der Chemischen Industrie and the EU Commission, Directorate XII, for financial support. $\mathrm{P}$. K. is grateful to the Cusanuswerk - Bischöfliche Hochbegabtenförderung - for a PhD fellowship.

\section{References}

(1) Stereoselective Synthesis of Steroids and Related Compounds, part VII. For part VI see: Groth, U.; Richter, N.; Kalogerakis, A. Eur. J. Org. Chem. 2003, 23, 4634.

(2) (a) Comprehensive Natural Products Chemistry, Vol. 8; Elsevier Science Ltd.: Amsterdam, 1999, 108-136. (b) Ernst, M.; Helmchen, G. Angew. Chem. Int. Ed. 2002, 41, 4054; Angew. Chem. 2002, 114, 4231. (c) Helmchen, G.; Gocke, A.; Lauer, G.; Urmann, M. Angew. Chem., Int. Ed. Engl. 1990, 29, 1024; Angew. Chem. 1990, 102, 1079(3).

(3) (a) Oppolzer, W.; Cunningham, F. Tetrahedron Lett. 1986, 26, 5467. (b) Mash, E. A. J. Org. Chem. 1987, 52, 4142. (c) Lawler, D. M.; Simpkins, N. S. Tetrahedron Lett. 1988, 29, 1207. (d) Suzuki, T.; Tada, H.; Unno, K. J. Chem. Soc., Perkin Trans. 1 1992, 2017. (e) Groth, U.; Halfbrodt, W.; Köhler, T.; Kreye, P. Liebigs Ann. Chem. 1994, 885. (f) Urban, E.; Knühl, G.; Helmchen, G. Tetrahedron 1995, 51, 13031.

(4) Helmchen, G.; Ihrig, K.; Schindler, H. Tetrahedron Lett. 1987, 28, 183 .

(5) (a) Fürstner, A.; Müller, T. Synlett 1997, 1010. (b) Fürstner, A.; Langenmann, K. J. Org. Chem. 1996, 61, 8746. (c) The Total Synthesis of Natural Products, Vol. 11; John Wiley \& Sons, Inc.: New York, 1994, 172-173. (d) Dactylol is a bicyclic sesquiterpene, which can be synthesized starting from a 2,3-disubstituted cyclopentanone.
(6) (a) Quinkert, G.; Müller, T.; Königer, A.; Schultheis, O.; Sickenberger, B.; Dürner, G. Tetrahedron Lett. 1992, 33, 3469. (b) The Total Synthesis of Natural Products, Vol. 11; John Wiley \& Sons, Inc.: New York, 1994, 148-150. (c) Confertin can also be synthesized from a 2,3disubstituted cyclopentanone.

(7) (a) Noyori, R.; Suzuki, M. Angew. Chem. Int. Ed. Engl. 1984, 23, 847; Angew. Chem. 1984, 96, 854. (b) Steglich, W.; Fugmann, B.; Lang-Fugmann, S. RÖMPP Natural Products; Thieme: Stuttgart, 2000, 517-518.

(8) (a) Wiechert, R. Angew. Chem. Int. Ed. Engl. 1970, 9, 321; Angew. Chem. 1970, 82, 331. (b) Wiechert, R. Angew. Chem. Int. Ed. Engl. 1970, 16, 506; Angew. Chem. 1977, 89, 513. (c) Quinkert, G.; Stark, H. Angew. Chem. Int. Ed. Engl. 1983, 22, 637; Angew. Chem. 1983, 95, 651. (d) Steglich, W.; Fugmann, B.; Lang-Fugmann, S. RÖMPP Natural Products; Thieme: Stuttgart, 2000, 608-611.

(9) (a) Yamamoto, Y. In Houben-Weyl, Methods of Organic Chemistry, Vol. E 21b; Thieme: Stuttgart, 1995, 20412155. (b) Urban, E.; Knühl, G.; Helmchen, G. Tetrahedron 1996, 52, 971. (c) Kanai, M.; Tomioka, K. Tetrahedron Lett. 1994, 35, 895. (d) Hailes, H. C.; Isaac, B.; Javaid, M. H. Tetrahedron Lett. 2001, 42, 7325. (e) Denmark, S. E.; Kim, J.-O. J. Org. Chem. 1995, 60, 7535. (f) Hanessian, S.; Gomtsyan, A.; Malek, N. J. Org. Chem. 2000, 65, 5623. (g) Hua, D. H.; Chan-Yu-King, R.; McKie, J. A.; Myer, L. J. Am. Chem. Soc. 1987, 109, 5026. (h) Barnhart, R. W.; Wang, X.; Noheda, P.; Bergens, S. H.; Whelan, J.; Bosnich, B. J. Am. Chem. Soc. 1994, 116, 1821. (i) Barnhart, R. W.; McMorran, D. A.; Bosnich, B. Chem. Commun. 1997, 589. (j) Moritani, Y.; Appella, D. H.; Jurkauskas, V.; Buchwald, S. L. J. Am. Chem. Soc. 2000, 122, 6797. (k) Liang, L.; Au-Yeung, T. T.-L.; Chan, A. S. C. Org. Lett. 2002, 4, 3799.

(10) (a) Nugent, W. A.; Hobbs, F. W. Jr. J. Org. Chem. 1983, 48, 5364. (b) Nugent, W. A.; Hobbs, F. W. Jr. Org. Synth. 1988, 66, 52. (c) Tietze, L. F.; Beifuss, U. Angew. Chem., Int. Ed. Engl. 1993, 32, 131; Angew. Chem. 1993, 105, 137. (d) Rossiter, B. E.; Swingle, N. M. Chem. Rev. 1992, 92, 771.

(11) (a) Groth, U.; Köhler, T.; Taapken, T. Tetrahedron 1991, 47, 7583. (b) Groth, U.; Huhn, T.; Richter, N. Liebigs Ann. Chem. 1993, 49. (c) Groth, U.; Köhler, T.; Taapken, T. Liebigs Ann. Chem. 1994, 665. (d) Groth, U.; Taapken, T. Liebigs Ann. Chem. 1994, 669.

(12) Sumitomo, H.; Kobayashi, K.; Saiji, T. J. Polym. Sci. 1972, 10,3421

(13) (a) Ort, O. Org. Synth. 1987, 65, 203. (b) Scharf, H.-D.; Buschmann, H. Synthesis 1988, 827; and references cited therein.

(14) Oppolzer, W.; Moretti, R.; Godel, T.; Meunier, A.; Löher, H. Tetrahedron Lett. 1983, 24, 4971. 
(15) (a) Lipshutz, B. H. Synlett 1990, 119. (b) Lipshutz, B. H. Org. React. 1992, 41, 135.

(16) Vinyllithium was prepared via reaction of tetravinyltin with $n$-BuLi.

(17) Experimental Procedure: A solution of $10.0 \mathrm{mmol}$ organolithium compound in $\mathrm{Et}_{2} \mathrm{O}(10 \mathrm{~mL})$ was added to a solution of $5.0 \mathrm{mmol}$ copper(I) cyanide in $\mathrm{Et}_{2} \mathrm{O}(10 \mathrm{~mL})$ at $-80{ }^{\circ} \mathrm{C}$. After $2 \mathrm{~h}$ stirring $5.0 \mathrm{mmol} \mathrm{BF} \cdot \mathrm{Et}_{2} \mathrm{O}$ were added and the resultant mixture was cooled at $-95^{\circ} \mathrm{C}$. A solution of 1.0 mmol chiral enoate 5 in $\mathrm{Et}_{2} \mathrm{O}(10 \mathrm{~mL})$ was added via canulla and the obtained mixture was allowed to warm under stirring to r.t. $(18 \mathrm{~h})$. The reaction mixture was quenched with aq sat. $\mathrm{NH}_{4} \mathrm{Cl}$ solution $(30 \mathrm{~mL})$, extracted with $\mathrm{Et}_{2} \mathrm{O}(2$ $\times 20 \mathrm{~mL}$ ), the combined organic layer dried over $\mathrm{MgSO}_{4}$ and evaporated in vacuum. Purification of the residue by flash chromatography provided the 2,3-substituted cyclopentanone 7 .

Analytical data of selected compounds (Figure 1).

Compound 7e: $\mathrm{R}_{\mathrm{f}} 0.41\left(\mathrm{Et}_{2} \mathrm{O}-\right.$ petroleum ether, 1:5). IR(film): 3040 (alkene $\mathrm{CH}), 1740(\mathrm{C}=\mathrm{O}), 1725(\mathrm{OC}=\mathrm{O})$, 1650 (alkene $\mathrm{C}=\mathrm{C}$ ), 1610 (arom. $\mathrm{C}=\mathrm{C}$ ) $\mathrm{cm}^{-1} .{ }^{1} \mathrm{H}$ NMR (250 $\left.\mathrm{MHz}, \mathrm{CDCl}_{3}\right): \delta=0.87\left(\mathrm{~d},{ }^{3} \mathrm{~J}=6.5 \mathrm{~Hz}, 1 \mathrm{H}, \mathrm{H}-5^{\prime}\right), 1.19(\mathrm{~s}, 3$ $\left.\mathrm{H}, \mathrm{CH}_{3}\right), 1.27$ (s, $\left.3 \mathrm{H}, \mathrm{CH}_{3}\right), 0.80-1.84$ (m, $7 \mathrm{H}, \mathrm{H}-1^{\prime}, \mathrm{H}-3^{\prime}$, H-4', H-6'), 1.88-2.40 (m, 3 H, H-4, H-5, H-2'), 3.46 (d, $\left.{ }^{3} J=11 \mathrm{~Hz}, 1 \mathrm{H}, \mathrm{H}-2\right), 2.83-3.05$ (m, $1 \mathrm{H}, \mathrm{H}-3$ ), 4.81 (ddd, $\left.{ }^{3} J=10.5,10.5,4 \mathrm{~Hz}, 1 \mathrm{H}, \mathrm{COOCH}, \mathrm{H}-1^{\prime}\right), 5.09\left(\mathrm{ddd}, J_{c i s}=\right.$ $\left.10 \mathrm{~Hz}, J=1.5,1.5 \mathrm{~Hz}, 1 \mathrm{H}, \mathrm{CH}=\mathrm{CH}_{2}\right), 5.125$ (ddd, $J_{\text {trans }}=17$ $\left.\mathrm{Hz}, J=1.5,1.5 \mathrm{~Hz}, 1 \mathrm{H}, \mathrm{CH}=\mathrm{CH}_{2}\right), 5.745$ (ddd, $J_{\text {trans }}=17$ $\left.\mathrm{Hz}, J_{c i s}=10 \mathrm{~Hz}, J=7 \mathrm{~Hz}, 1 \mathrm{H}, \mathrm{CH}=\mathrm{CH}_{2}\right), 7.06-7.20(\mathrm{~m}, 1$ $\mathrm{H}$, arom. $\mathrm{H}), 7.23-7.38$ (m, $4 \mathrm{H}$, arom. $\mathrm{H}) .{ }^{13} \mathrm{C}$ NMR (62.5 $\left.\mathrm{MHz}, \mathrm{CDCl}_{3}\right): \delta=21.74$ [22.75] $\left(\mathrm{CH}_{3}\right), 25.68\left(\mathrm{CH}_{3}\right), 26.58$ (cyclopentane- $\mathrm{CH}_{2}$ ), [26.43] 26.65 (cyclohexane- $\mathrm{CH}_{2}$ ), 27.37 [27.51] $\left(\mathrm{CH}_{3}\right),[29.52] 31.28\left(\mathrm{CHCH}_{3}, \mathrm{C}-5^{\prime}\right), 34.52$ [34.68] (cyclohexane- $\mathrm{CH}_{2}$ ), 37.79 [38.25] (cyclopentane$\left.\mathrm{CH}_{2}\right), 39.70$ [39.87] $\left(\mathrm{C}\left(\mathrm{CH}_{3}\right)_{2}\right), 41.38$ [41.73] (cyclohexane$\left.\mathrm{CH}_{2}\right), 43.73$ [46.20] $\left(\mathrm{CHCH}=\mathrm{CH}_{2}, \mathrm{C}-3\right), 49.96$ [50.48] (C2'), 60.90 [62.07] (COCHCO, C-2), 75.96 [76.30] (C-1'), 115.78 [116.24] $\left(\mathrm{CH}=\mathrm{CH}_{2}\right), 124.87$ [125.18], 125.45 [125.56], [127.84] 127.97 ( $3 \times$ C-arom.), 138.62 [140.67] $\left(\mathrm{CH}=\mathrm{CH}_{2}\right), 151.51$ (C-arom.), 167.34 [168.13] (COO), 210.22 (CO) (signals of the $2 R, 3 S$-configured diastereomer in brackets). EI-MS $(70 \mathrm{eV}): \mathrm{m} / \mathrm{z}(100)=119\left[\mathrm{PhC}\left(\mathrm{CH}_{3}\right)_{2}\right]$ (100), 249 (4) $\left.\left[\mathrm{M}^{+}-\mathrm{PhC}\left(\mathrm{CH}_{3}\right)_{2}\right)\right], 368$ (2) [ $\left.\mathrm{M}^{+}\right]$. Anal. Calcd for $\mathrm{C}_{24} \mathrm{H}_{32} \mathrm{O}_{3}$ (368.5): $\mathrm{C}, 78.22 ; \mathrm{H}, 8.75$. Found: $\mathrm{C}, 78.39 ; \mathrm{H}$, 8.85 .

Compound 7f (Table 1, entry 11): $\mathrm{R}_{\mathrm{f}} 0.46\left(\mathrm{Et}_{2} \mathrm{O}-\right.$

petroleum ether, $1: 1$ ); $\mathrm{mp}: 59-63{ }^{\circ} \mathrm{C}$. IR(nujol): 3060,3040 (arom. $\mathrm{CH}), 1750(\mathrm{C}=\mathrm{O}), 1730(\mathrm{OC}=\mathrm{O}), 1640(\mathrm{C}=\mathrm{C}), 1610$, 1595 (arom. $\mathrm{C}=\mathrm{C}), 1350,1165\left(\mathrm{CSO}_{2} \mathrm{~N}\right) \mathrm{cm}^{-1} .{ }^{1} \mathrm{H}$ NMR $\left(250 \mathrm{MHz}, \mathrm{CDCl}_{3}\right): \delta=0.82\left(\mathrm{~s}, 3 \mathrm{H}, \mathrm{CH}_{3}\right), 0.88(\mathrm{~s}, 3 \mathrm{H}, \mathrm{C}-$ $\left.1-\mathrm{CH}_{3}\right), 1.04\left(\mathrm{~s}, 3 \mathrm{H}, \mathrm{CH}_{3}\right), 0.90-2.45\left(\mathrm{~m}, 9 \mathrm{H}, \mathrm{CH}, \mathrm{CH}_{2}\right)$, $2.02\left(\mathrm{~s}, 3 \mathrm{H}\right.$, arom. $\left.\mathrm{CH}_{3}\right), 2.32\left(\mathrm{~s}, 3 \mathrm{H}\right.$, arom. $\left.\mathrm{CH}_{3}\right), 3.33$ (d, $\left.J_{\text {trans }}=11.2 \mathrm{~Hz}, 1 \mathrm{H}, \mathrm{H}-2^{\prime}\right), 3.34-3.50\left(\mathrm{~m}, 1 \mathrm{H}, \mathrm{H}-3^{\prime}\right), 4.25$ (ddd, $J=8.8 \mathrm{~Hz}, J=3.4 \mathrm{~Hz},{ }^{4} J=1.0 \mathrm{~Hz}, 1 \mathrm{H}, \mathrm{H}-3$ ), 5.11 (ddd, $J_{\text {cis }}=10.6 \mathrm{~Hz},{ }^{2} J=1.2 \mathrm{~Hz},{ }^{4} J=1.2 \mathrm{~Hz}, 1 \mathrm{H}, \mathrm{CH}=\mathrm{CH}_{2}$ ), 5.27 (ddd, $J_{\text {trans }}=17.0 \mathrm{~Hz},{ }^{2} J=1.2 \mathrm{~Hz},{ }^{4} J=1.2 \mathrm{~Hz}, 1 \mathrm{H}$, $\left.\mathrm{CH}=\mathrm{CH}_{2}\right), 5.46(\mathrm{~d}, J=8.8 \mathrm{~Hz}, 1 \mathrm{H}, \mathrm{H}-2), 5.78(\mathrm{~s}, 1 \mathrm{H}$, arom. $\mathrm{H}), 5.98\left(\mathrm{ddd}, J_{\text {trans }}=17.0 \mathrm{~Hz}, J_{\text {cis }}=10.6 \mathrm{~Hz}, J=6.6 \mathrm{~Hz}, 1\right.$ $\left.\mathrm{H}, \mathrm{CH}=\mathrm{CH}_{2}\right), 6.83(\mathrm{~s}, 1 \mathrm{H}$, arom. $\mathrm{H}), 7.11(\mathrm{~s}, 1 \mathrm{H}$, arom. $\mathrm{H})$, 7.28-7.57 (m, $5 \mathrm{H}$, arom. $\mathrm{H}) .{ }^{13} \mathrm{C} \mathrm{NMR}\left(62.5 \mathrm{MHz}, \mathrm{CDCl}_{3}\right)$ : $\delta=14.30$ (C-10), 19.41 (C-8), 19.51 (C-9), 19.69 (C-5), 20.98, $21.29\left(2 \times\right.$ arom. $\left.\mathrm{CCH}_{3}\right), 26.59(\mathrm{C}-6), 26.62\left(\mathrm{C}-4^{\prime}\right)$, 38.13 (C-5'), [35.53] 43.46 (C-3'), 45.71 (C-7), 49.38 (C-4), 51.30 (C-1), 59.35 (C-3), 60.77 [63.27] (C-2'), 77.59 (C-2),
$115.38\left(\mathrm{CH}=\mathrm{CH}_{2}\right), 127.49,128.12,129.33,129.89,132.46$ (arom. C), $138.64\left(\mathrm{CH}=\mathrm{CH}_{2}\right), 136.98,137.05,138.16$, 139.04 (arom. C), 167.88 (CHCOO), 210.70 [212.35] (C=O) (signals of the $2^{\prime} R, 3^{\prime} S$-configured diastereomer in brackets). EI-MS $(70 \mathrm{eV}): \mathrm{m} / \mathrm{z}(\%)=549(3)\left[\mathrm{M}^{+}\right], 395(11)\left[\mathrm{M}^{+}-\right.$ $\left.\mathrm{C}_{8} \mathrm{H}_{10} \mathrm{O}_{3}\right], 254$ (100) $\left[\mathrm{M}^{+}-\mathrm{C}_{8} \mathrm{H}_{10} \mathrm{O}_{3}-\mathrm{SO}_{2} \mathrm{C}_{6} \mathrm{H}_{5}\right], 105$ (22) $\left[\mathrm{C}_{8} \mathrm{H}_{9}^{+}\right]$. Anal. Calcd for $\mathrm{C}_{32} \mathrm{H}_{39} \mathrm{O}_{5} \mathrm{NS}$ (549.7): C, 69.92; H, 7.15. Found: C, 69.87; H, 7.28.

Compound 8e (Table 2, entry 5): $\mathrm{R}_{\mathrm{f}} 0.31\left(\mathrm{Et}_{2} \mathrm{O}-\right.$-petroleum ether, 1:2). $[\alpha]_{\mathrm{D}}{ }^{20}+82.3\left(c 1.5, \mathrm{CHCl}_{3}\right) . \mathrm{Bp} .65-70^{\circ} \mathrm{C}(2$ torr). IR(film): 3065 (alkene $\mathrm{CH}), 1750(\mathrm{C}=\mathrm{O}), 1730$ $(\mathrm{OC}=\mathrm{O}), 1635(\mathrm{C}=\mathrm{C}) \mathrm{cm}^{-1} .{ }^{1} \mathrm{H}$ NMR $\left(250 \mathrm{MHz}, \mathrm{CDCl}_{3}\right)$ : $\delta=1.54-1.86(\mathrm{~m}, 1 \mathrm{H}, \mathrm{CH}), 2.16-2.60\left(\mathrm{~m}, 3 \mathrm{H}, \mathrm{CH}, \mathrm{CH}_{2}\right)$, $3.03\left(\mathrm{dd},{ }^{3} J=11.6 \mathrm{~Hz},{ }^{4} J=0.6 \mathrm{~Hz}, 1 \mathrm{H}, \mathrm{C}-2-\mathrm{H}\right), 3.14-3.29$ $(\mathrm{m}, 1 \mathrm{H}, \mathrm{C}-3-\mathrm{H}), 3.76\left(\mathrm{~s}, 3 \mathrm{H}, \mathrm{OCH}_{3}\right), 5.10\left(\mathrm{ddd},{ }^{3} J_{\text {cis }}=10.0\right.$ $\left.\mathrm{Hz},{ }^{2} J=1.2 \mathrm{~Hz},{ }^{4} J=1.2 \mathrm{~Hz}, 1 \mathrm{H}, \mathrm{CH}=\mathrm{CH}_{2}\right), 5.17$ (ddd, $\left.{ }^{3} J_{\text {trans }}=17.0 \mathrm{~Hz},{ }^{2} \mathrm{~J}=1.2 \mathrm{~Hz},{ }^{4} \mathrm{~J}=1.2 \mathrm{~Hz}, 1 \mathrm{H}, \mathrm{CH}=\mathrm{CH}_{2}\right)$, $5.84\left(\mathrm{ddd},{ }^{3} J_{\text {trans }}=17.0 \mathrm{~Hz},{ }^{3} J_{\text {cis }}=10.0 \mathrm{~Hz},{ }^{3} J=6.6 \mathrm{~Hz}, 1 \mathrm{H}\right.$, $\left.\mathrm{CH}=\mathrm{CH}_{2}\right) \cdot{ }^{13} \mathrm{C}$ NMR $\left(62.5 \mathrm{MHz}, \mathrm{CDCl}_{3}\right): \delta=27.25(\mathrm{C}-4)$, 38.12 (C-5), $44.83(\mathrm{C}-3), 52.48\left(\mathrm{OCH}_{3}\right), 60.77(\mathrm{C}-2), 115.95$ $\left(\mathrm{CH}=\mathrm{CH}_{2}\right), 135.95\left(\mathrm{CH}=\mathrm{CH}_{2}\right), 169.10\left(\mathrm{COOCH}_{3}\right), 210.71$ $(\mathrm{C}-1)$. EI-MS $(70 \mathrm{eV}): \mathrm{m} / \mathrm{z}(\%)=168(86)\left[\mathrm{M}^{+}\right], 137(85)$ $\left[\mathrm{M}^{+}-\mathrm{OCH}_{3}\right], 109(90)\left[\mathrm{M}^{+}-\mathrm{COOCH}_{3}\right], 81(100)\left[\mathrm{M}^{+}-\right.$ $\mathrm{C}_{2} \mathrm{H}_{3} \mathrm{O}_{2}-\mathrm{C}_{2} \mathrm{H}_{3}-\mathrm{H}$ ]. HRMS: $\mathrm{m} / z$ calcd for $\mathrm{C}_{9} \mathrm{H}_{12} \mathrm{O}_{3}(168.2)$ : 168.0786; found: 168.0786 .

Compound 10e (Table 2, entry 5): $\mathrm{R}_{\mathrm{f}} 0.41\left(\mathrm{Et}_{2} \mathrm{O}-\right.$ petroleum ether, 1:10). $[\alpha]_{\mathrm{D}}{ }^{20}+3.3\left(c 1.4, \mathrm{CHCl}_{3}\right)$. IR(film): 3065 (alkene $\mathrm{CH}), 1635(\mathrm{C}=\mathrm{C}) \mathrm{cm}^{-1}$. ${ }^{1} \mathrm{H}$ NMR $(250 \mathrm{MHz}$, $\left.\mathrm{CDCl}_{3}\right): \delta=1.21-1.27\left(\mathrm{~m}, 6 \mathrm{H}, \mathrm{CH}_{3}\right), 1.31-2.08(\mathrm{~m}, 6 \mathrm{H}$, $\left.\mathrm{CH}_{2}\right), 2.46-2.74\left(\mathrm{~m}, 1 \mathrm{H}, \mathrm{CHCH}=\mathrm{CH}_{2}\right), 3.47-3.66(\mathrm{~m}, 2 \mathrm{H}$, $\mathrm{OCH}), 4.91\left(\mathrm{ddd},{ }^{3} J_{\text {cis }}=10.0 \mathrm{~Hz},{ }^{2} J=1.5 \mathrm{~Hz},{ }^{4} J=1.5 \mathrm{~Hz}, 1\right.$ $\mathrm{H}, \mathrm{CH}=\mathrm{CH}_{2}$ ), $5.00\left(\mathrm{ddd},{ }^{3} J_{\text {trans }}=17.2 \mathrm{~Hz},{ }^{2} J=1.5 \mathrm{~Hz},{ }^{4} \mathrm{~J}=\right.$ $\left.1.5 \mathrm{~Hz}, 1 \mathrm{H}, \mathrm{CH}=\mathrm{CH}_{2}\right), 5.79\left(\mathrm{ddd},{ }^{3} J_{\text {trans }}=17.2 \mathrm{~Hz},{ }^{3} J_{\text {cis }}=\right.$ $\left.10.0 \mathrm{~Hz},{ }^{3} \mathrm{~J}=7.2 \mathrm{~Hz}, 1 \mathrm{H}, \mathrm{CH}=\mathrm{CH}_{2}\right) \cdot{ }^{13} \mathrm{C} \mathrm{NMR}(62.5 \mathrm{MHz}$, $\left.\mathrm{CDCl}_{3}\right): \delta=16.86\left(\mathrm{CH}_{3}\right), 16.95\left(\mathrm{CH}_{3}\right), 30.57,37.98\left(\mathrm{CH}_{2}\right)$, $42.16(\mathrm{C}-7), 44.43\left(\mathrm{CH}_{2}\right), 78.21\left(\mathrm{CHCH}_{3}\right), 78.26\left(\mathrm{CHCH}_{3}\right)$, $113.07\left(\mathrm{CH}=\mathrm{CH}_{2}\right), 116.84(\mathrm{OCO}), 141.98\left(\mathrm{CH}=\mathrm{CH}_{2}\right)$. EIMS (70 eV): $m / z(\%)=182(3)\left[\mathrm{M}^{+}\right], 114(100)\left[\mathrm{C}_{6} \mathrm{H}_{10} \mathrm{O}_{2}{ }^{+}\right]$, 54 (26) $\left[\mathrm{C}_{4} \mathrm{H}_{8}{ }^{+}\right]$. Anal. Calcd for $\mathrm{C}_{11} \mathrm{H}_{18} \mathrm{O}_{2}$ (182.3): C, 72.49; $\mathrm{H}, 9.95$. Found: $\mathrm{C}, 72.36$; H, 9.84 .

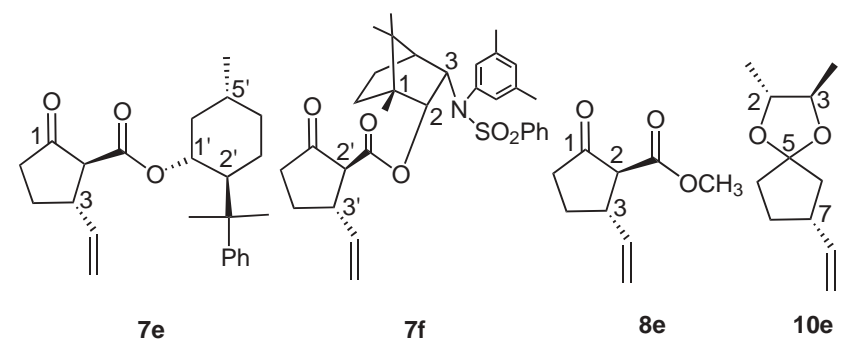

Figure 1

(18) Marx, J. N.; Norman, L. R. J. Org. Chem. 1975, 40, 1602.

(19) (a) For 9b $(\mathrm{R}=\mathrm{Ph}):[\alpha]_{\mathrm{D}}{ }^{20}=+83.4\left(c 0.3, \mathrm{CHCl}_{3}\right)$. (b) Taber, D. F.; Raman, K. J. Am. Chem. Soc. 1983, 105, 5935. (c) Taura, Y.; Tanaka, M.; Wu, X.-M.; Funakoshi, K.; Sakai, K. Tetrahedron 1991, 47, 4879.

(20) For $9 \mathrm{c}(\mathrm{R}=t-\mathrm{Bu}):[\alpha]_{\mathrm{D}}{ }^{20}=+134.8\left(\mathrm{c} 1.13, \mathrm{CHCl}_{3}\right)$; ref. ${ }^{19 \mathrm{~b}}$

(21) Hiemstra, H.; Wynberg, H. Tetrahedron Lett. 1977, 25, 2183.

(22) (a) Parish, E. J.; Mody, N. V.; Hedin, P. A.; Miles, D. H. J. Org. Chem. 1974, 39, 1592. (b) Huang, B.-S.; Parish, E. J.; Miles, D. H. J. Org. Chem. 1974, 39, 2647. 